\title{
AN AUDIT OF PAIN SCORES AND PONV RATES IN THE PACU AND WARD FOLLOWING
}

DIFFERENT TYPES OF BREAST SURGERY

\section{H. Abrahams ${ }^{1}$.}

${ }^{1}$ Nobles Hospital, Anaesthesia and Critical Care, Douglas, Isle of Man.

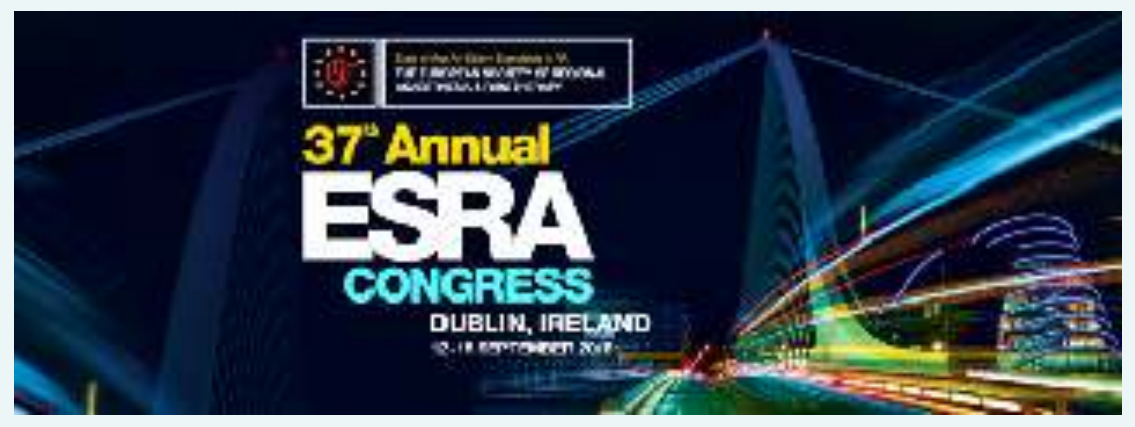

Background and Aims:

Pectoral Nerve (PECS) and Serratus Plane Blocks (SPB) have become popular alternatives to established regional anaesthesia techniques for breast surgery since first described in 2011 and 2013 respectively.

These blocks are easy to learn and administer to patients under ultrasound guidance and appear to reduce post-operative pain scores/opioid consumption as well opioid-related adverse effects (PONV).

I investigated the qualitative performance of these blocks with reference to pain scores and PONV episodes in the PACU/general ward.

Methods:

Electronic case notes for 25 patients undergoing breast surgery between September 2017 and April 2018 were retrospectively reviewed.Local audit/IRB approval was obtained. All patients had consented to having either a pectoral and/or a serratus plane nerve block sited under ultrasound guidance following GA.Postoperative pain scores (NRS) and PONV episodes requiring treatment both in the PACU and general ward were recorded.

\section{$\underline{\text { Results }}$}
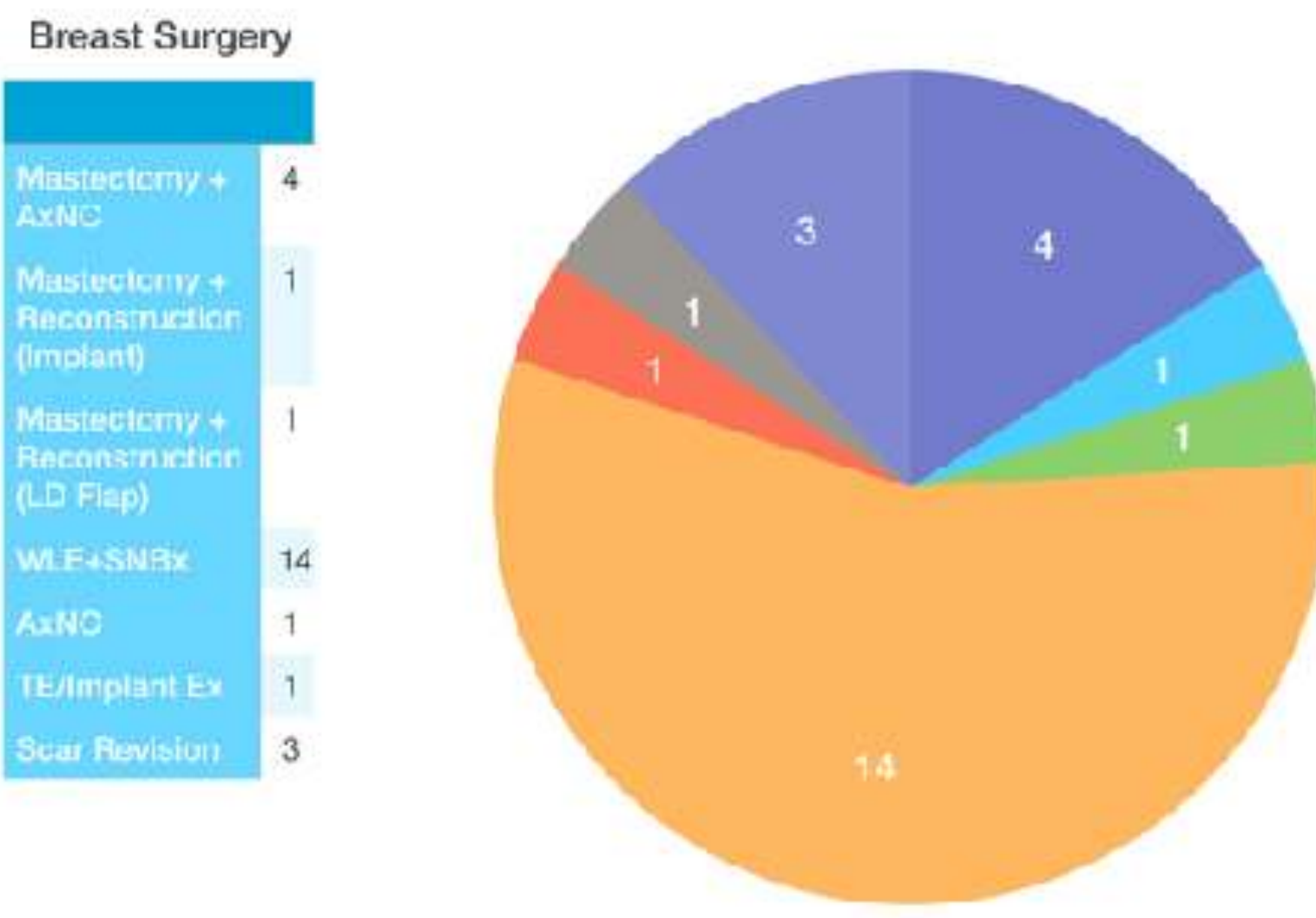

- Mastectomy + AxhC

Mastectomy + Reccrstruction (Implant)

Mastectemy + Roconstruction (LD Flap)

WLLE+SNEx

AxNC

TE/mplant Ex

Scar devision
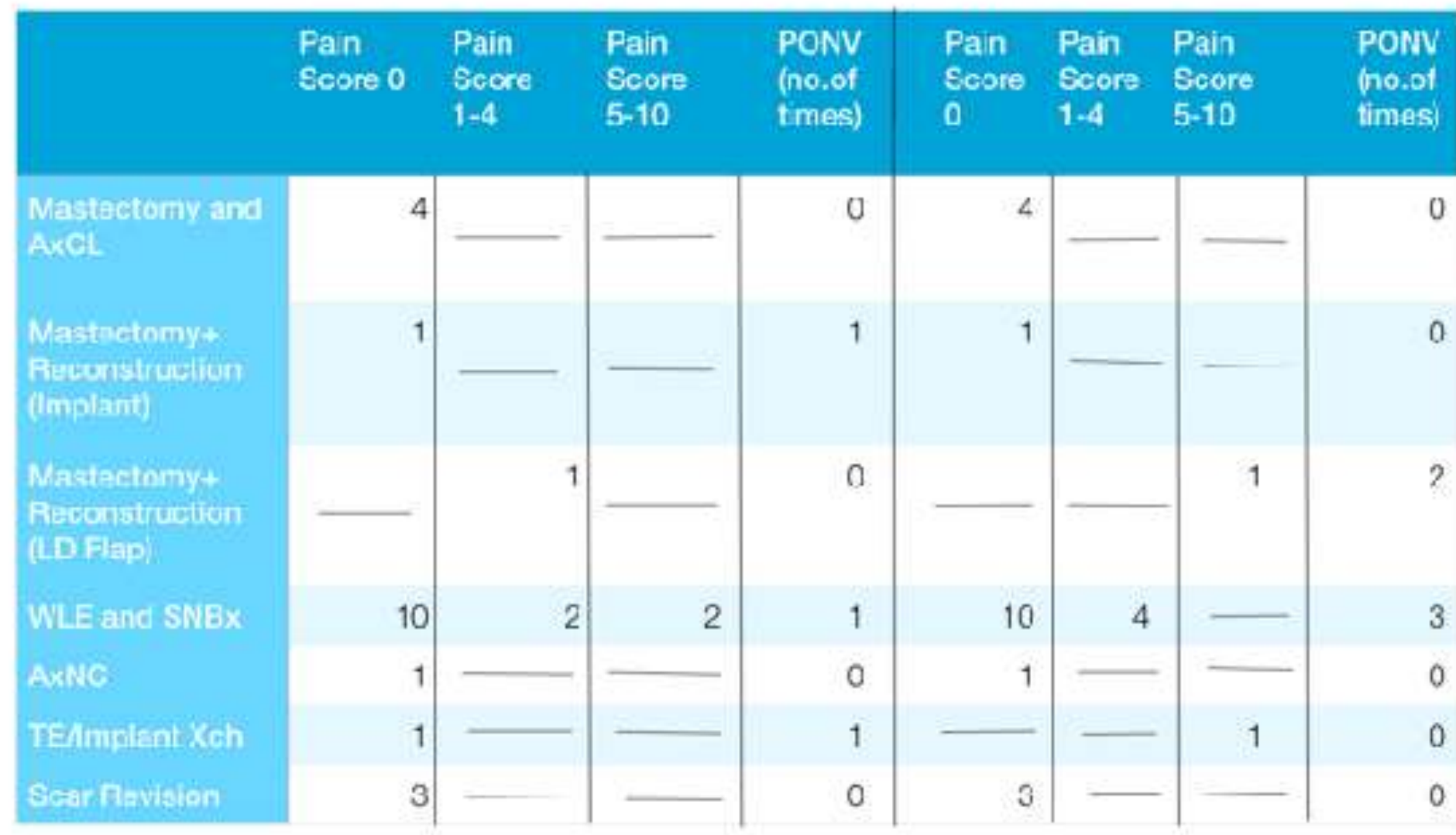

PACU : Pain Score 0/10 $80 \%$ cases

PONV - 3 episodes

$12 \%$ cases

WARD : Pain Score 0/10

$76 \%$ cases

PONV - 5 episodes

$20 \%$ cases

The majority of patients had a pain score of $0 / 10$ both in the PACU $(86 \%)$ and 24 hours later in the general ward(76\%).The 2 cases with high PACU pain scores were both WLE with SNBX - this suggests block failure.This was not translated into a similar pain score on the ward 24 hours later.There was an increased number of PONV episodes in these same patients on the ward. $12 \%$ of patients had PONV requiring treatment in the PACU, $20 \%$ in the ward.

\section{Conclusions:}

The results are generally encouraging with a high success rate as evidenced by low PACU/ward pain scores as well as low PONV events requiring treatment. Future research should investigate possible impact on chronic pain following breast surgery. 